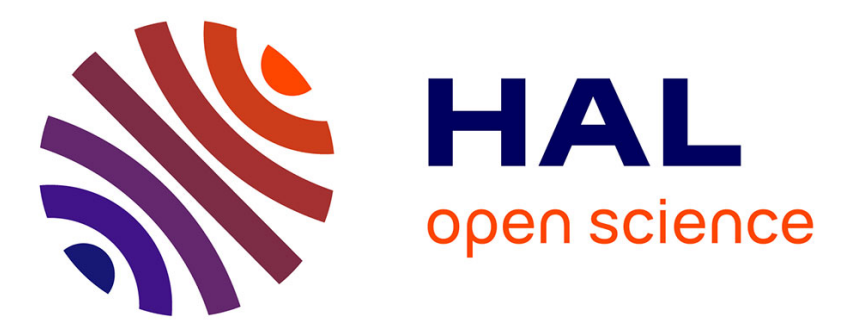

\title{
The AMBRE ILE: how to use Case-Based Reasoning to teach methods
}

\author{
Nathalie Guin, Stéphanie Jean-Daubias, Sandra Nogry
}

\section{To cite this version:}

Nathalie Guin, Stéphanie Jean-Daubias, Sandra Nogry. The AMBRE ILE: how to use Case-Based Reasoning to teach methods. ITS 2002: 6th International Conference, Jun 2002, Biarritz, France. pp.782-791. hal-01574296

\section{HAL Id: hal-01574296 https://hal.science/hal-01574296}

Submitted on 12 Aug 2017

HAL is a multi-disciplinary open access archive for the deposit and dissemination of scientific research documents, whether they are published or not. The documents may come from teaching and research institutions in France or abroad, or from public or private research centers.
L'archive ouverte pluridisciplinaire HAL, est destinée au dépôt et à la diffusion de documents scientifiques de niveau recherche, publiés ou non, émanant des établissements d'enseignement et de recherche français ou étrangers, des laboratoires publics ou privés. 


\title{
The AMBre ILE: How to Use Case-Based Reasoning to Teach Methods
}

\author{
Nathalie Guin-Duclosson, Stéphanie Jean-Daubias and Sandra Nogry \\ Laboratoire d'Ingénierie des Systèmes d'Information \\ Université Claude Bernard - Lyon 1 \\ Nautibus, 8 bd Niels Bohr, Campus de la Doua \\ 69622 Villeurbanne Cedex \\ FRANCE \\ \{nguin, sdaubias, snogry\}@bat710.univ-lyon1.fr
}

\begin{abstract}
Designing an Interactive Learning Environment (ILE) requires work within a multi-disciplinary team [9]. In this paper, we describe the designing process of the ILE AMBRE, the purpose of which is to teach methods. We show how, based on didactic work, we chose to represent the expert knowledge to be taught to the learner through Artificial Intelligence techniques. We also describe how we adjusted the Case-Based Reasoning paradigm (CBR), derived from Artificial Intelligence, to teaching abstract knowledge based on problem classes. Last we describe a Cognitive Psychology experiment we conducted in order to test the appropriateness of the thus designed EIAH to the goals of the AMBRE project.
\end{abstract}

\section{Introduction}

This paper describes the multidisciplinary work conducted in the framework of the AMBRE project. The purpose of the project is to complete an Interactive Learning Environment (ILE) to teach methods. Derived from didactic studies, these methods are based on a classification of problems and solving tools.

In order to teach abstract knowledge, we suggest to use the Case-Based Reasoning paradigm (CBR). Our theory is that the use of cases already experienced by the learner to solve problems and the comparison between experienced cases and problems to be solved can help the learner acquire abstract knowledge (schemata, problem classes).

In the first part of this paper, we describe the knowledge that we would like the learner to acquire through the AMBRE ILE, and how we represent it within an Artificial Intelligence system. We then present how such expertise can be connected with CBR, and describe existing work regarding ILE using CBR. In a third section, we show the teaching process, which the AMBRE project is based on, as well as the first ILE prototype implemented. We finish with a description of an experiment intended to test the appropriateness of the prototype to the goals of the project, the results of the experimentation leading us to express recommendations for designing the AMBRE ILE. 


\section{How to Represent the Knowledge to Be Taught?}

Didactic studies suggest teaching explicitly the methods, which, in a small domain, help to guide the problem solving process [14][15]. These methods are based on a classification of problems and of solving tools. They help select the solving technique that is best suited to a given problem.

As part of the AMBRE project, we consider relying on a problem solver to help the learning, particularly in order to provide the learner with explanations. The problem solver should therefore work according to the methods it wishes to have acquired, not according to the most general expert methods of the domain [4]. Thus, the solver should be designed and derived from the targeted knowledge.

In order to represent such knowledge, we use the SYRCLAD [7] solver architecture which enables to explicit in a declarative way a problem classification and the reformulation and solution knowledge associated with it. Thus it enables to model the knowledge to be observed in learners, not only the knowledge of an expert.

In a given domain, an expert (didactician, teacher...) defines a hierarchy of problem classes. In order to be able to use that hierarchy to classify a problem, an expert should define the reformulation knowledge that allows computing the values of discriminating attributes of the classification hierarchy. Some classes in the hierarchy, referred to as operational, are specific enough for each one to be associated with a solving technique suited for the problems in that class (according to the system).

Solving a problem then consists first of all in an operationalisation phase, where the system uses the reformulation knowledge and the domain problem classification graph to determine what class the problem is in and to build a new model of that problem (referred to as operational model). This model is mainly composed of the discriminating attributes of the hierarchy, but also of attributes which distinguish the problem from its class. This operational model no longer has surface features (i.e. problem elements irrelevant to the solution) of the descriptive model. The solving in itself then consists in applying the solving technique associated with the class of problem to the operational model, in order to get a solution to the posed problem.

The purpose of the AMBRE project is to design an ILE the purpose of which is to have the learner acquire a solving method based on a problem class hierarchy and on solving techniques associated with those classes. However, it does not always seem appropriate to present such a hierarchy to the learner explicitly. In some domains, the terms defining solving techniques and classes are not necessarily used institutionally and are unknown to the learners. Therefore we consider a process where the learner is more active, where he or she builds his own method, by representing a class of problem by a prototype problem. We believe that the case-based reasoning paradigm can help the learner acquire such a method. 


\section{Use Case-Based Reasoning}

\subsection{From SYRCLAD to CBR}

Case-Based Reasoning can be described as a set of sequential steps (elaborate, retrieve, adapt, test/revise, store) that is often represented by a cycle [13]. We liken such a cycle (steps in bold type in Figure 1) to a solving method implemented with the SYRCLAD architecture (steps in italics type in Figure 1).

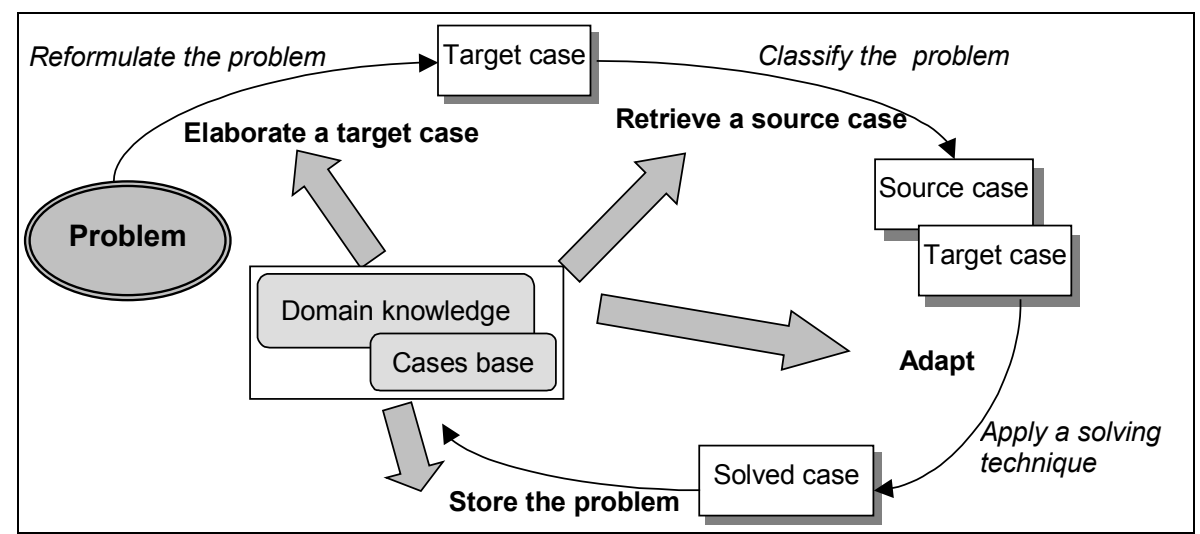

Fig. 1. SYRCLAD and the CBR cycle

The problem reformulation step can be seen as a target case elaboration step, even if that step is then advanced since it uses classification knowledge and reformulation knowledge. The problem classification can also be matched with a source case retrieval step, the retrieved source case then constituting the prototype case of the problem class that a SYRCLAD solver would have determined. Adapting the solving of that source case to the target case is then like applying the solving technique to the operational class represented by the prototype. In order to store the solved problem, one can consider that the SYRCLAD solver connects the new case with a problem class. The SYRCLAD solver does not use the revision step of usual CBR cycle, since the solving is always correct. In the AMBRE ILE, revision is included in each step of the cycle, as it is explained in part 4.

Our objective is to have acquired a solving method based on a problem class hierarchy by presenting examples of solved problems to the learner, and guiding the solution of new problems using case-based reasoning. We wish the " expert-student » knowledge after the learning can be modelled like in Figure 2: based on a cases base, the learner has built operational classes represented by a prototype problem, and these problem classes can be organised in a hierarchy. 


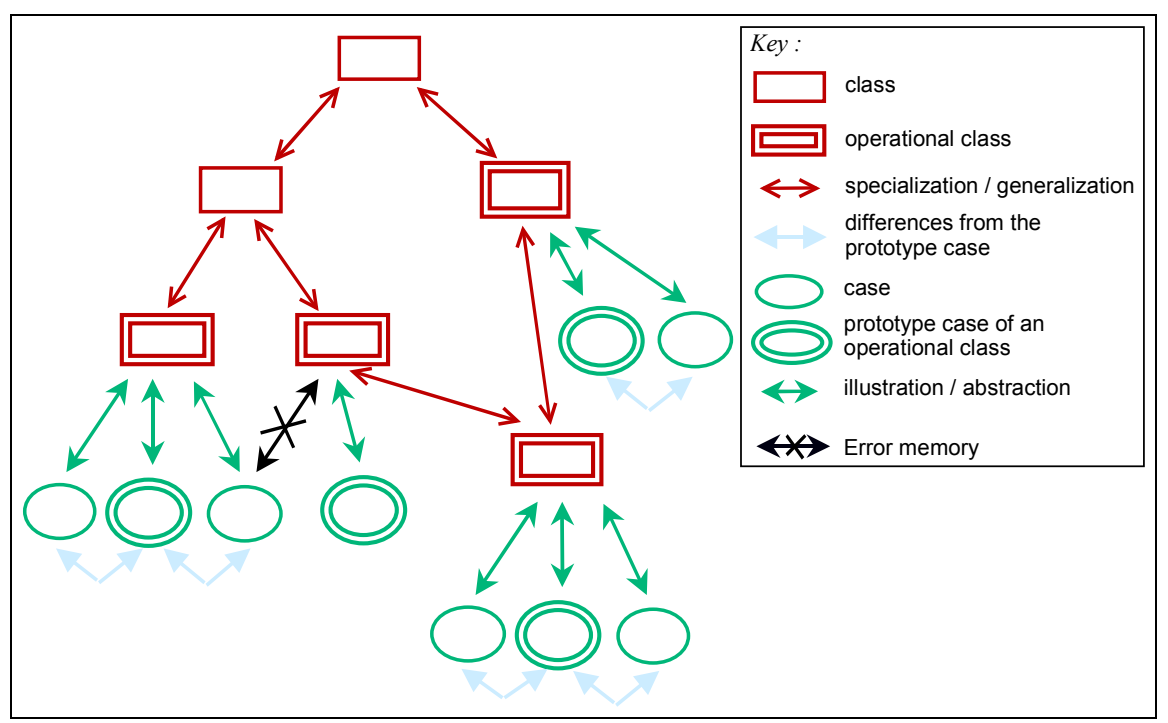

Fig. 2. "Expert-Student" knowledge model

\subsection{CBR Uses in ILE}

The CBR paradigm is a technique that has already been used in various parts of an ILE.

Modelling the learner's knowledge can be done using CBR [18], like the assessment of the leaner [10]. By comparing the learner's model with other learners' assessments (forming a cases base), CBR can also help select a learning strategy [5], or build a path in a hypertext [8].

The closest application of our theory is Case-Based Teaching [17]. Systems based on this learning strategy present a close case to the learner when the leaner is having difficulty solving a problem, or when facing a problem he or she never came across before (in a new domain or a new type). In these systems there are several levels of interactivity between the learner and the computer environment [19]. The learner can ask the system to find a similar example and explain how that case was solved; the system can also offer the entire solution to the learner's exercise like in CATO [1].

The Virtual Participant [12] is an example of case-based teaching. It is a virtual assistant intervening in virtual lectures to present cases to the learners. The cases base includes problems posed by persons taking part in the lecture, and solutions (offered by the same persons). This environment has been tried in a course where forum type lectures were given. From one year to the other, the participants experienced similar problems; thus the Virtual Participant helps meet their expectations more efficiently by offering similar cases experiences by previous year students. 


\section{The Learning Principle in AMBrE}

In the case of the AMBRE project, the purpose was to have the learner acquire a solving method for problems in one domain. To do so, the first step is to present him, in an interactive manner, the solving of a few typical problems (serving as cases base initialisation). The learner is then assisted in solving new problems (usually in the same class as the submitted examples): the environment guides the learner's solving of the problem by following each step of the CBR cycle (see Figure 3). Elaboration, retrieval, adaptation and storage are done by the learner, but guided by the system.

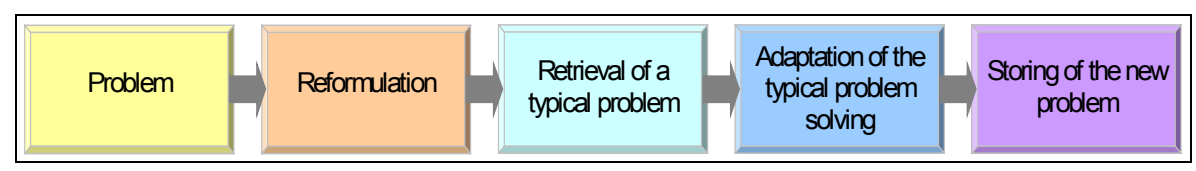

Fig. 3. The solving steps in AMBRE

\subsection{Elaboration of the Target Case}

The first step consists in reformulating the problem to be solved. The learner is asked to build a new formulation of the submitted problem, step by step. Actually this task consists in identifying the structural features of the problem to be solved (i.e. relevant features for the solution, as opposed to surface features), by finding attributes of the operational problem model. The system relies on the classification graph to guide the reformulation. It reviews the student's answers and explains possible mistakes in order to reach an agreement with the student on a correct reformulation. The reformulation (operational model) no longer has most of the initial problem's surface features, and becomes a reference for the remainder of the solving.

\subsection{Retrieval of a Source Case}

The second step of the solving consists in the learner comparing the problem to be solved with those he or she has already solved by identifying differences and similarities in each case. He or she should choose the prototype problem (source case) that seems the nearest to the problem to be solved, such nearness being based on reformulated problems, not on surface features. By choosing a prototype exercise, the learner implicitly identifies the class of problems associated with the problem to be solved. The system diagnoses the student's proposal and may suggest and justify a better choice, in order to reach an agreement with the student on the reference problem. 


\subsection{Adaptation of the Source Case Solution to the Target Case}

In order to complete the solving, the learner should adapt the solution of the source case (the reference problem) to the target case (the problem to be solved). At this point, we are hopeful that little by little, the learner will relate a solving plan to the problem class. The system prepares such an adaptation by outlining differences and similarities between the source case and the target case. When both cases belong to the same operational class, adapting the solution of the prototype problem comes to applying the solving technique associated with its respective class to the values of the significant attributes of the problem to be solved (those that distinguish the problem from the class it is an instance of). The solving technique, known to the system, is not explicitly known to the learner. That is why the learner should rely on the source case solution and adapt it to the target case.

It can be considered that in a first phase, the system will show the learner how to carry out the adaptation step with an example, in order to introduce the interface tools helping to achieve the adaptation. When the learner puts forward an adaptation resulting in a solution proposal, the system diagnoses the proposal and may provide explanations on corrections to be made in order to reach a correct solution.

\subsection{Storage of the Target Case}

Once the problem is solved, the learner should add it to the cases base, i.e. insert it in the problems structure he or she has built. During that phase, the learner should identify, explicitly this time, the problem class associated with the problem to be solved. This task will often consists in relating the new case to a prototype problem, therefore in inserting it in a group of existing problems. A group of problems includes all problems in the same operational class. Obviously the learner should be given an opportunity to change the selection of the prototype problem representing a group. In addition, it may be advisable that the prototype problems all belong to the same family of problems (in the meaning of surface features), so as to better focus on structure differences.

It may occur that the new case does not belong to an existing group of problems, and the learner can then create a new group whose prototype problem is the problem he or she has just solved. It may also occur that one (or several) sub-groups of problem need to be created within a group, the sub-group being also represented by a prototype problem. The purpose is to represent an operational class, sub-class of another operational class; to the sub-class is related a more specific solving technique than the one related to the parent class.

Last, the learner should be given tools to help him, if he or she wishes, distinguish problem groups from one another, by building an initial hierarchy. To do so, it is advisable that he or she uses attributes of operational models, which he or she is familiar with through the problem reformulation step. 


\section{Prototype Evaluation}

A prototype of the AMBRE ILE was made (see Figure 4 for the retrieval step) for the numbering problem domain (final scientific year level) ${ }^{1}$. The prototype reproduces the operation of the system with a limited number of problems, as the Artificial Intelligence modules of the systems have not been integrated yet. Indeed, for the AMBRE environment to provide the functions described in the above section, conventional ILE modules, i.e. the expert module, the learner's model, the diagnosis module, the educational module and the aid and explanation module should be completed and the SYRCLAD solver integrated.

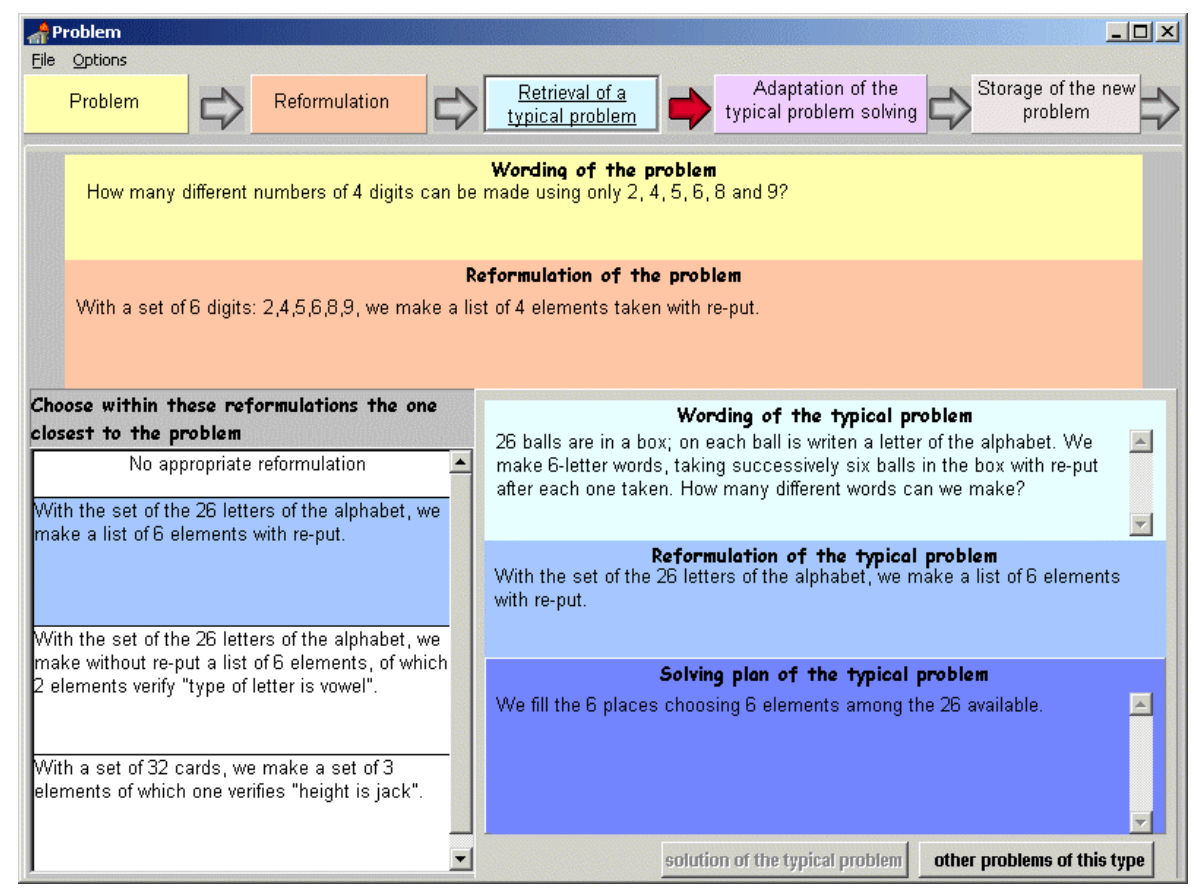

Fig. 4. The prototype retrieval step

Prior to developing the entire ILE, we wanted to assess the impact of the CBR paradigm on method learning by observing, at first, the behaviour of users with the prototype.

As part of this preliminary evaluation, conducted according to cognitive psychology techniques, two hypotheses were tested:

- the use of CBR paradigm facilitates the induction of problems categories,

- the use of the CBR paradigm helps improve the learner's performance by problem solving.

Below, we describe the experiment conducted and the results obtained.

\footnotetext{
${ }^{1}$ The problem class hierarchy for this domain was determined as part of the "Combien?" work group, composed of Artificial Intelligence researchers and mathematic teachers [6].
} 


\subsection{Experimentation}

This first evaluation consists of a comparison study of the use of two prototypes; the first prototype guides the solving according to the ILE AMBRE principle, and therefore integrates the CBR paradigm. The second prototype offers, as a control condition, a simple solving of the same exercises in the same environment, but without any special guiding.

Before and after the training with the prototype (see Figure 5), the learners solve two paper-pencil tasks:

- a problem solving task helping to measure the learners' performance,

- a problem categorisation task (classification of ten exercises wordings based on solution similarity) providing indication of learners' classification.

The experiment was conducted with 64 students in their final scientific year, in the classroom in order to reproduce actual use conditions.

The tasks offered in pre-test and post-test were analysed and compared. For the categorisation task, the classifications given by the learners were subjected to a descriptive analysis using the Barthélémy and Guénoche taxonomy [2]. For the problem-solving task, the answers were processed using a variance analysis.

Prototype use traces were also analysed in order to observe software use conditions and determine most frequent errors.

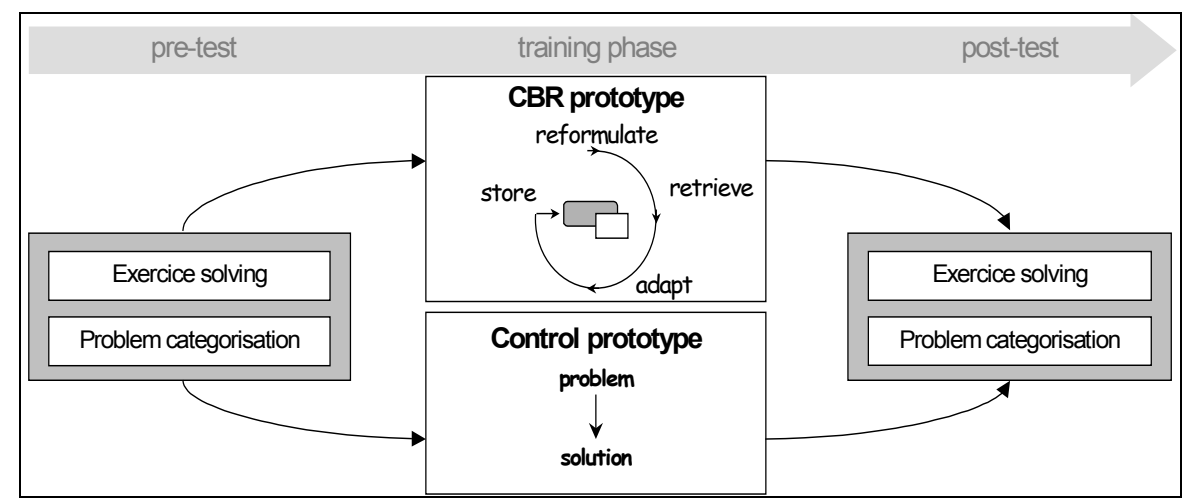

Fig. 5. Experimentation plan

\subsection{Results and Discussion}

First of all the results of this first experiment show that the prototype using the CBR paradigm facilitates problem classifying according to relevant features for the solving, but only with some learners. Then, during the post-test, there is no significant problem solving performance difference between both groups (those using the CBR prototype and those using the control prototype). In addition, use traces outline the difficulties experienced by the learners in finding the closest source exercise in the retrieval step; they often proceed by trial-error. Thus, even if the reformulation step helps explain structure features, it does not facilitate the use of such features in the retrieval step. 
Therefore these results do not validate our hypotheses (problem category induction facilitation and improved problem solving performance). One could assume these disappointing results come from the difficulty experienced by some learners during the retrieval step (the way we presented this step on the interface should be improved). These results are also partly due to the lack of system flexibility, which does not allow the learner to circulate between the various process steps.

\subsection{Advice for Designing the ILE}

The results of the experiment underline the need to improve the ILE prototype before carrying on with the tests. The retrieval step seems the most important one to develop. There should be more incentives to compare the target problem and the typical problems. To do so, we should offer tools to the learner facilitating such comparison. We have already imagined the techniques which in the next version of the prototype, will help outline the similarities and differences between the problem to be solved and the case base derived problems. In addition, transition from one step to the other should be made possible so the learner may understand the impact of the choice of the typical problem on the remainder of the resolution (the adaptation step), and so he or she can relate the retrieval and storage step. One can assume that by offering greater freedom for movement between CBR steps, the learner's awareness of the relationship between such steps will be made easier. The integration of artificial intelligence modules in the prototype should add flexibility, particularly for this point.

\section{Conclusions and Prospects}

This paper described the AMBRE ILE project, which relies on the CBR solving cycle to have the learner acquire a problem solving method based on a classification of problems in the domain. For this study, we designed and implemented a prototype of the ILE using the CBR paradigm to facilitate method learning by guiding the student towards problem classification and solving tools. Thus we took into account, in addition to the CBR paradigm, cognitive psychology studies that demonstrate the importance of using examples when elaborating abstract knowledge [3]. In order to assess the impact of this paradigm on problem classification and solving tools, we set up an experiment comparing the prototype to a prototype without CBR.

Although the results of this first cognitive evaluation do not confirm our hypotheses, they give a better understanding of the learner's behaviour and views. This enables us to express recommendations that should make easier, in the AMBRE ILE, the learner's classification of problems and solving tools. The new prototype, integrating at least part of the artificial intelligence modules, and taking into account the recommendations expressed after the first experiment, should help us set up new experiments with a view to assessing the impact of the CBR on learning problem classes and on learning solving tool classes. A longitudinal study would also be useful to observe the learner's problem classification as time passes. Last, additional experiments on learning from examples will help provide a better understanding of how to induce some learning processes, and thereby help method teaching. 


\section{References}

1. Aleven, V., Ashley, K.D.: Teaching Case-Based Argumentation through a Model and Examples - Empirical Evaluation of an Intelligent Learning Environment. Artificial Intelligence in Education (B. du Boulay and R. Mizoguchi Eds.), IOS Press (1997) 87-94

2. Barthélemy, J.P., Guénoche, A.: Trees and Proximity Representations. Wiley J., London (1991) 238

3. Cummins, D.: Role of Analogical Reasoning in the Induction of problem categories. Journal of Experimental Psychology: Learning, Memory, and Cognition, Vol. 5 (1992) 1103-1124

4. Delozanne, E.: Explications en EIAO : études à partir d'ÉLISE, un logiciel pour s'entraîner à une méthode de calcul de primitives. Phd, Université du Maine, Le Mans, France (1992)

5. Gilbert, J.E.: Case-Based Reasoning Applied to Instruction Method Selection for Intelligent Tutoring Systems. Workshop 5: Case-Based Reasoning in Intelligent Training Systems, ITS'2000, Montreal (2000) 11-15

6. Guin, N., Giroire H., Tisseau G.: Le classement de problèmes : une méthode de résolution de problèmes pour le module expert d'un EIAO. Application aux problèmes de dénombrement. In proceedings of "Quatrièmes journées EIAO de Cachan" (Guin, D., Nicaud, J.-F, Py, D. Eds.), Eyrolles, France (1995) 113-124

7. Guin-Duclosson, N.: SYRCLAD: une architecture de résolveurs de problèmes permettant d'expliciter des connaissances de classification, reformulation et résolution. Revue d'Intelligence Artificielle, vol 13-2, Paris : Hermès (1999) 225-282

8. Héraud, J.-M., Mille, A.: Pixed: vers le partage et la réutilisation d'expériences pour assister l'apprentissage. In Proceedings of international symposium TICE 2000, Troyes, France (2000) 237-244

9. Jean, S.: PÉPITE: un système d'assistance au diagnostic de compétences, Phd, Université du Maine, Le Mans, France (2000)

10. Khan, T.M.: Case-Based Evaluation for Student Modelling. Workshop 5: Case-Based Reasoning in Intelligent Training Systems, ITS'2000, Montreal (2000) 16-22

11. Kolodner, J.: Case Based Reasoning. San Mateo, CA: Morgan Kaufmann Publishers (1993)

12. Masterton, S.: The Virtual Participant: Lessons to be Learned from a Case-Based Tutor's Assistant. Computer Support for Collaborative Learning, Toronto (1997)

13. Mille, A.: Associer expertise et expérience pour assister les tâches de l'utilisateur, Habilitation à diriger des recherches, Université Claude Bernard, Lyon, France (1998)

14. Rogalski, M.: Enseigner des méthodes en mathématiques. Commission Inter-Irem Université, Enseigner autrement les mathématiques en Deug A première année, bulletin Inter-Irem (1990) 65-79

15. Rogalski, M.: Les concepts de l'EIAO sont-ils indépendants du domaine? L'exemple d'enseignement de méthodes en analyse. Recherches en Didactiques des Mathématiques, vol $14 n^{\circ} 1.2$ (1994) 43-66

16. Sander, E.: L'analogie, du naïf au créatif. Paris, l'Harmattan (2000)

17. Schank, R., Edelson, D.: A Role for AI in Education: Using Technology to Reshape Education. Journal of Artificial Intelligence in Education vol 1.2 (1990) 3-20

18. Shiri, A., Aimeur, E., Frasson, C.: SARA: A Cased-Based Student Modelling System. Fourth European Workshop on Case-Based Reasoning, Lecture Notes in Artificial Intelligence, $\mathrm{n}^{\circ} 1488$, Dublin, Ireland (1998) 425-436

19. Tourigny, N., Capus, L.: Towards Making Intelligent Training Systems Using Examples more Flexible and Reusable by Exploiting Case-Based Reasoning. Workshop 5: Case-Based Reasoning in Intelligent Training Systems, ITS'2000, Montreal (2000) 23-28 